\title{
LA DEFENSA DE LOS DERECHOS HUMANOS ECONÓMICOS Y SOCIALES Y LOS LÍMITES DE LA INTERVENCIÓN JUDICIAL
}

\author{
Osvaldo Guariglia \\ Consejo Nacional de Investigaciones Científicas y Técnicas (Buenos Aires)
}

RESUMEN. La cuestión de la implementación de los derechos humanos, especialmente los económicos y sociales, se ha convertido en uno de los puntos más controvertidos de la filosofía política y jurídica actual. La creación de la Corte Penal Internacional puso de manifiesto el status especial de los derechos humanos civiles y políticos que se sitúan por encima de las soberanías nacionales. Los derechos económicos y sociales, sin embargo, están lejos de haber alcanzado una situación similar. En el presente trabajo discutiré la tradicional distinción entre derechos negativos y positivos, el papel del estado en general y de los jueces en especial en el resguardo de ambos tipos de derechos y mostraré las correlaciones que existen entre ambos tipos de derechos. Analizaré, por último, de qué modo es factible que los jueces defiendan la validez y vigencia de los derechos humanos económicos y sociales cuando los otros poderes, ejecutivo y legislativo, no ofrecen una efectiva implementación de aquellos. Esta intervención del poder judicial es usualmente considerada una indebida intromisión de los jueces en la arena democrática, sin tener tras de sí el respaldo de haber sido elegidos por la ciudadanía. Intentaré responder a esta objeción.

Palabras clave: Derechos humanos económicos y sociales, protección estatal y judicial.

ABSTRACT. The issue of human rights implementation, especially that of economic and social human rights, has become one of the most controversial subjects in current legal and political philosophy. The establishment of the International Criminal Court revealed the special status of civil and political human rights as standing above national sovereignties. Economic and social rights, however, are far from reaching a similar position. In this paper I will discuss the traditional distinction between negative and positive rights, as well as the role of the state in general and that of the judiciary in particular in the protection of both kinds of rights; and I will show the correlations between both types of rights. Lastly, I will analyse in which way it is feasible that judges defend the validity and efficacy of social and economic rights when the other branches of government, the executive and the legislative, do not offer an effective implementation of them. This intervention of the judiciary is usually seen as an inappropriate interfering of judges in the democratic arena, without the backing of having been elected by the citizenry. I will try to answer to this objection.

Keywords: social and economic human rights, state and judicial protection. 


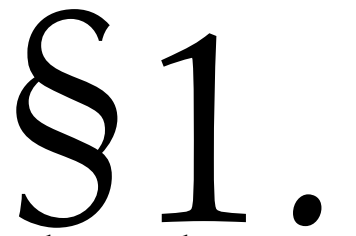

Las relaciones entre moral y derecho son tan estrechas como controvertidas desde antiguo. Tanto los intentos de unificar ambos campos distintos en uno y el mismo, provenientes de visiones metafísicas, religiosas o ideológicas del mundo, como los antagónicos de cortar drásticamente todo lazo entre ambos, usualmente a cargo de corrientes positivistas extremas, se han demostrado incapaces de obtener una aprobación amplia de juristas y filósofos. Parece indudable que, tratándose de dos disciplinas normativas referidas a la conducta personal y social, la moral y el derecho necesariamente tendrán que tener varios puntos de contacto en los rasgos que los caracterizan. Por comenzar, los juicios de ambos son enunciados en la misma clase de lenguaje, el prescriptivo; luego, ambos se refieren a las acciones humanas y comparten ambos, por último, una misma modalidad, la deóntica. ¿Qué es, pues, lo que los distingue? Podemos aceptar por el momento la caracterización que propone KANT, a saber: lo que diferencia al derecho es «la facultad de coaccionar a quien lo viola», de modo tal que la misma razón que impone al agente la obligación de actuar según una ley universal, lo exime de la exigencia interior de hacerlo por una elección moral propia (MS, Ak. VI, 231). KANT parte de varios supuestos que no es necesario compartir para aceptar la distinción que propone salvo uno: que tanto la moral como el derecho están fundados en razones, por lo cual las tareas de cada una de estas disciplinas son entre sí complementarias. Compartir este supuesto sigue siendo uno de los rasgos distintivos del pensamiento filosófico, tanto moral como jurídico y político, que se asume a sí mismo dentro de la corriente inaugurada por el gran ilustrado.

La Declaración Universal de los Derechos Humanos de 1948 ha introducido un nuevo punto de contacto pero también de roce entre moral y derecho, que a su vez ha puesto en marcha una discusión aún en curso sobre la especificidad, la validez universal y la implementación de esos proclamados derechos. Al ser considerados derechos morales, ¿debe admitirse por ello que se trata de derechos anteriores a toda inclusión de los mismos en una constitución histórica o en tratados internacionales y, en consecuencia, de «derechos que la sociedad civil no acuerda sino que reconoce y sanciona como universalmente válidos» (MARITAIN, 1953: p. 89, véase también FINNIS, 1980: pp. 198 y ss.)? ¿O, al contrario, se trata desde un comienzo de derechos exclusivamente jurídicos que el constituyente incorpora como derechos subjetivos fundamentales dentro de una democracia cuyos procedimientos institucionales deben ser regulados por aquellos para ser considerados legitimos (HABERMAS, 2002: p. 201)? Sin duda, entre las dos posiciones opuestas que he presentado a modo de hitos orientadores existen intentos de tender un puente entre ambas concepciones, sobre todo porque hay buenas razones para sostener tanto la una como la otra. En efecto, como argumentaba NiNO, el carácter de derechos morales, externos a toda legislación positiva, aparece claro allí donde los derechos básicos no son ni respetados ni reconocidos (NINO, 1989: p. 48, n. 37). La cuestión se ha hecho aún más controvertida y acuciante a partir de la publicación del libro de John RAWLS, The Law of Peoples (1999), que provocó una polémica todavía en curso sobre el alcance y la validez de los derechos humanos en el campo internacional. En discusión no hay una sino dos alternativas en juego que tienen que ver con los derechos humanos en su conjunto: I) ¿Son los derechos humanos proclamados en la Declaración Universal y ratificados luego por sendas Convenciones sólo la expresión de pricipios éticos propios de un estado 
democrático liberal o son efectivamente derechos universales que pertenecen a todos los individuos del planeta, independientemente de la sociedad, la cultura y el régimen político en que vivan? Y II) ¿Tienen los derechos civiles y políticos, que aseguran las libertades básicas de los individuos y sus derechos de asociación y participación política, el mismo nivel que los derechos económicos y sociales que no solamente protegen la actividad privada de los miembros de una sociedad civil sino que también les confieren ciertos derechos básicos de subsistencia, salud y seguridad social?

El tema que me propongo tratar atraviesa todas esas cuestiones, las que sería imposible exponer aquí en detalle, de modo que me limitaré a presentar una tesis general sobre los puntos más controvertidos para discutir luego con mayor detalle la cuestión central que me ocupa: si la intervención de los jueces en la salvaguarda de los derechos humanos económicos y sociales es una injustificada intromisión de un poder aristocrático en la esfera propia de los poderes democráticamente elegidos o es, en cambio, una irrenunciable función que les cabe en resguardo de las reglas básicas de un sistema democrático.

$\$ 2$. Adoptaré, en primer término, una distinción que RAWLS ha propuesto al precisar el rol que desempeñan los derechos humanos en la teoría ideal del derecho internacional. Como es sabido, RAWLS distingue una subclase de derechos humanos, los comprendidos por los arts. 3 al 18 de la Declaración, de todos los demás, considerándolos un núcleo básico que funciona como un criterio universal para todos los pueblos: «su fuerza política (moral) se extiende a todas las sociedades y son obligatorios para todos los pueblos y sociedades, incluidos los estados fuera de la ley» (RAWLS, 1999: 80). Las sociedades no liberales que respetan este núcleo básico, aun cuando ellas posean y ejerzan una única idea comprensiva de la buena vida y tengan una organización política jerárquica limitada con un sistema sólo "decente" de consulta, deben ser consideradas miembros legítimos de la Sociedad de los Pueblos y deben ser excluidos de toda intervención externa fundada en su carencia de respeto por algunos de los derechos humanos propios de una sociedad democrática (véase la discusión de BEITZ, 2001: pp. 274 y ss.). Es evidente que, de esta manera, los derechos humanos dejan de ser un conjunto de principios provinciales de las democracias liberales y pasan a ser principios universales efectivos sin tener que apelar para ello a interpretaciones hermenéuticas forzadas de tradiciones religiosas densas a fin de extraer de éstas por vía analógica algunos principios sustantivos más o menos similares a aquellos contenidos en los derechos humanos propiamente dichos (contra WALZER, 1994, passim). $\mathrm{Al}$ mismo tiempo, es también palmario que los derechos humanos como tales no tienen el mismo rol ni el mismo sentido en las democracias liberales que en las sociedades jerárquicas decentes. Para estas últimas, en efecto, son estándares de evaluación impuestos desde fuera del propio sistema cultural, jurídico y político a guisa de criterios morales a los que estos puebls debieron adaptar sus anteriores usos y costumbres, sin perjuicio de que este ethos tradicional pudiera admitir con mayor o menor dificultad tal adaptación (véase la esclarecedora discusión de COHEN, 2004: pp. 190-213). Mediante los pactos y convenios internacionales, los regímenes políticos jerárquicos que se incorporan a la Sociedad de los Pueblos admiten una restricción de su soberanía política y una mayor laxitud de sus concepciones religiosas y culturales fundadas en el monopolio del espacio público para dar cabida, dentro de ciertos límites, a otras formas minoritarias de culto, y a una cierta igualdad de todos sus súbditos ante la ley. Estas 
concesiones a los principios morales universales contenidos en los derechos humanos dan prueba de la buena voluntad de los pueblos jerárquicos decentes en su concepción religiosa o metafísica del bien común en el ámbito doméstico y del respeto por los demás seres humanos como tales en el ámbito internacional, con lo que ponen de manifiesto su vocación pacifista, pero al mismo tiempo demuestran que sus concepciones políticas públicas están muy distantes de la concepción democrática y liberal de justicia.

Para una democracia constitucional, en cambio, los derechos humanos son intrínsecos al régimen, fueron proclamados conjuntamente con las reglas básicas de funcionamiento del sistema y desempeñan un rol fundamental en la marcha sin obstáculos de éste. En efecto, todo régimen republicano y democrático está cimentado en dos principios básicos de justicia que establecen la igualdad y la libertad de todos los ciudadanos como partícipes e integrantes de una misma sociedad política. Dado que son reglas constitutivas del régimen democrático, tales principios no pueden ser considerados anteriores y externos al régimen mismo sino que establecen las condiciones sine qua non de relaciones simétricas y recíprocas entre sus ciudadanos que son definitorias de una democracia (véase HABERMAS, 1997: pp. 223-225; 277 y ss.). Los artículos que van del primero al vigésimo primero de la Declaración Universal de los DDHH incluyen el conjunto de derechos fundamentales que están contenidos en todas las constituciones democráticas desde fines del siglo XVIII en adelante. Se trata, por cierto, de los ahora conocidos como derechos civiles y políticos que enuncian todos aquellos derechos negativos de los cuales goza cada ciudadano como simple partícipe del régimen constitucional. En su conjunto le garantizan no solamente la protección de su vida sino también el ejercicio de su libertad de pensamiento, de culto, de expresión, de asociación no sólo a fin de ejercer una profesión o comercio lícito sino también de ejercer el derecho a participar en los cargos y oficios públicos sin sufrir discriminaciones por religión, sexo, raza o clase social.

Ahora bien, desde el artículo vigésimo segundo al artículo vigésimo octavo la Declaración incluye otros derechos, los así llamados derechos económicos y sociales, que se fueron incorporando a las constituciones liberales a lo largo del siglo Xx y que son considerados derechos positivos, cuyo estatus filosófico y jurídico está fuertemente controvertido por una corriente filosófica libertaria que niega su naturaleza de derechos, frente a otra corriente, liberal igualitaria, que sostiene enfáticamente su carácter de derechos tan legítimos como los primeros. Pese a que estos derechos de segunda generación, como también se los conoce, fueron ratificados igualmente por una Convención especial en diciembre de 1966, continúan sin ser efectivamente implementados en la mayoría de los países en desarrollo — para no hablar de los países pobres- y están siendo frontalmente atacados por los gobiernos neo-conservadores de los países desarrollados. A fin de precisar los términos de la argumentación, señalaré antes de seguir avanzando algunos de los puntos críticos que hacen de los derechos humanos económicos y sociales un blanco preferido tanto de ataques devastadores como de pretextos excusadores de su incumplimiento.

$\$ 3$. En primer lugar es necesario señalar un aspecto en la distinción entre deberes negativos y positivos que, si bien ha sido reiteradamente señalada, no es siempre tenida debidamente en cuenta. Me refiero al hecho de que, en tanto obligaciones, ambas especies de deberes tienen la misma fuerza coercitiva. La diferencia entre éstas 
se basa, más bien, en el contenido material de la obligación: en el primer caso describe con precisión una especie de acciones que el agente está obligado a evitar, sin que pueda haber gradaciones en el cumplimiento de la obligación, ya que se trata de dejar solamente que continúe la misma situación de los otros agentes anterior a la omisión. En el caso de las obligaciones positivas, en cambio, el contenido de la obligación está constituido por un fin distinto al transcurso natural del estado de cosas previo a la acción, fin, por tanto, que sólo puede ser vagamente indicado, ya que tanto su ejecución como su resultado estarán condicionados en cada caso por las peculiaridades de la situación. Una característica adicional de esta división la constituye el alcance de cada tipo de deber: mientras que los negativos son casi exclusivamente deberes generales, los positivos son casi exclusivamente deberes especiales, lo que implica que es necesario que el sujeto de estos últimos satisfaga algún requisito previo (para una discusión más extensa remito a GUARIGLIA, 2002: pp. 123 y ss.).

Dejando de lado, pues, este aspecto, es necesario establecer que no hay una correlación estricta entre derechos y deberes del mismo signo, como sostiene el libertarismo. En efecto, los derechos negativos tienen como correlatos no solamente deberes negativos generales, en el sentido de que todos estamos obligados a respetar la vida y la integridad de las demás personas, sino también deberes positivos especiales, que afectan a los funcionarios y dirigentes gubernamentales, quienes tienen la obligación de prever y llevar a cabo todas las medidas necesarias de prevención y de represión de las lesiones que puedan cometerse o se cometan contra el derecho negativo de los sujetos titulares del mismo. Ahora bien, la existencia del reclamo por la seguridad e integridad de las personas proviene originalmente de un derecho negativo general, que está sin duda estrictamente correlacionado con un deber negativo perfecto también de carácter general, al que se agrega el deber positivo especial de los funcionarios públicos y de los jueces encargados de velar por la seguridad e integridad de las personas. El derecho positivo a que se resguarde mediante los poderes públicos la seguridad e integridad de la vida de los ciudadanos constituye, en consecuencia, un derecho positivo complementario y derivado del derecho negativo general, que es el que originalmente fundamenta el reclamo. En conclusión, si bien la distinción metódica entre derechos negativos y positivos debe ser asumida como conceptualmente fundada, el entretejido de derechos tanto negativos como positivos entre sí demuestra que en la implementación de los derechos, éstos se combinan para asegurar la efectiva protección de sus titulares, de modo que no es posible satisfacer los unos, en este caso los negativos, sin reconocer simultáneamente la existencia de otros, en este caso, los derechos positivos de protección de la seguridad de cada cual (cp. SHUE, 1996: pp. 37-40, GEWIRTH, 1996: pp. 34-38).

$\$ 4$. Consideremos ahora los derechos positivos originales, es decir, basados en un reclamo del sujeto de derecho, cuyo fin es la satisfacción de una necesidad básica. Prima facie se presentan dos serios interrogantes para la adecuada comprensión de estos derechos y, por consiguiente, su posterior implementación: determinar quiénes son los directamente afectados, tanto (a) en lo que concierne a los requisitos para ser sujetos beneficiarios de ese derecho como $(b)$ en lo que concierne a los parámetros mediante los que se debe definir quiénes habrán de asumir la carga de la obligación.

La primera cuestión, esto es, (a) quiénes son los afectados en su calidad de sujetos de derechos positivos, puede resolverse en general respondiendo que, potencial- 
mente, lo son todos los miembros de una misma sociedad, sea por poseer el derecho de ciudadanía dentro del estado correspondiente, sea por ser un residente extranjero legalmente admitido en éste. Ahora bien, dada la limitación de los recursos escasos, es necesario establecer una jerarquía entre los posibles beneficiarios, para lo cual será necesario seleccionar como criterio un conjunto mínimo de necesidades básicas que deben ser satisfechas. Contra esta última afirmación se ha observado con acierto que es imposible establecer por anticipado la magnitud y el carácter de las necesidades básicas en cada sociedad, ya que sobre ambas variables influyen de un modo decisorio tanto la cultura como el grado de desarrollo económico y tecnológico de la misma. Sin embargo, se puede señalar un indicador lo suficientemente preciso pero flexible para establecer una medida de las necesidades básicas, incluyendo dentro de ellas las de nutrición, habitación, educación y salud. Me refiero al conjunto de necesidades promedio requeridas para que un individuo de ambos sexos alcance un dominio suficiente de sus capacidades y habilidades como para poder asegurarse el ejercicio de su autonomia (Cp. GUARIGLIA, 1993: pp. 109-123, y 1996: pp. 173 y ss.; GEWIRTH, 1996: pp. 51 y ss.; FABRE, 1998: pp. 267 y ss.; O’NEILL, 2000: pp. 29-49, 137-138). La magnitud de los recursos necesarios para asegurar ese mínimo dependerá fuertemente del grado de desarrollo social al que haya alcanzado no solamente una misma nación, sino también las distintas regiones dentro de ésta. De esta manera, quienes caigan por debajo de ese mínimo, fijado según parámetros regionales y hasta comunales, por no hallarse en condiciones de satisfacer mediante sus propios medios las necesidades de subsistencia — sea por incapacidades temporarias o ermanentes, por desempleo temporáneo o crónico, etc.- deberán ser los beneficiarios prioritarios de los derechos económicos y sociales.

Más compleja se presenta la segunda cuestión, a saber: (b) quiénes deben asumir la carga de suministrar los recursos para satisfacer las necesidades de otros. Es, fundamentalmente, contra esta posibilidad que se levantan los libertarios, aduciendo que sería un modo de utilizar a ciertas personas, los sujetos a la obligación, como medios y no como fines en sí mismos (NozicK, 1980: pp. 32-33). A primera vista, se trata de una extraña objeción, puesto que echa mano a un argumento kantiano con el fin de oponerse a toda forma de solidaridad entre los ciudadanos de una misma sociedad. En su forma más elemental a lo que apunta es a denunciar una distribución desigual de las cargas, por ejemplo a través de los impuestos directos, cuyas tasas aumentan proporcionalmente a los mayores ingresos de cada uno, dado que en ese caso parecería haber una discriminación negativa en contra de los más ricos, cuando en realidad, por tratarse de un derecho universal, su costo debería ser también universalmente enjugado. Sin embargo, la objeción cae de inmediato cuando uno advierte que este deber positivo de contribuir al mantenimiento del estado — que es el que, en última instancia, distribuirá los recursos públicos- recae de un modo u otro en todos los miembros de la sociedad, y afecta tanto al mantenimiento del aparato policial y de seguridad indispensable para proteger los derechos negativos, como a los aportes para la seguridad social, la educación o la salud. En otros términos, la cuestión no reside en las cargas que hay que imponer para el sostenimiento del estado, pues éstas deberán existir en cualquier caso, sino en la amplitud y calidad de los servicios que el estado debe aportar a la sociedad, que es en definitiva lo que le importa al libertarismo, dada su insistencia en reducir al mínimo los bienes públicos provistos por aquél. Desde esta 
perspectiva, la objeción ya no se sostiene tal como fue formulada, pues ahora se ha desplazado a la jerarquía de prioridades que cada estado democrático, en uso d su soberanía, se da como fines de su acción política.

Considerada, entonces, desde el punto de vista de las obligaciones, la combinación entre deberes negativos y positivos es análoga a la de derechos negativos y positivos, dado que todo miembro de un determinado estado tendrá un deber negativo general tanto de no lesionar la seguridad de los demás como de no contribuir mediante las propias acciones a empeorar la situación ya de por sí precaria de los peor situados (por ejemplo, mediante explotaciones que deterioren la calidad del agua o del suelo, o por medio de prácticas corruptas que incidan en el deterioro de los servicios prestados por el estado en sus distintos niveles). A ello se añade un deber positivo especial para cada individuo, que consiste en cumplir con la carga que le corresponde de acuerdo con su nivel relativo de ingresos a fin de sostener al estado, de modo que este último cuente con los recursos necesarios tanto para mantener la seguridad de la población como para solventar las necesidades básicas de los peor situados.

$\$ 5$. Estamos en condiciones de abordar nuestro tema central, a saber, la compleja cuestión del cumplimiento y la efectiva ejecución de los derechos económicos y sociales y el rol que desempeñan en ello los jueces. En primer lugar, es necesario determinar el primer destinatario de las demandas nacidas como provenientes de esos derechos humanos, sin cuya identificación precisa amenazan con tornarse derechos meramente declamatorios. No cabe duda de que se trata de una obligación que debe asumir en cada caso el estado a través de sus diferentes niveles, desde el más amplio, nacional o federal, hasta los niveles regionales, provinciales y municipales o comunales. En efecto, se puede postular la existencia de deberes especiales que afectan a los gobernantes y funcionarios políticos del estado como correlato de los derechos positivos generales que asisten a cada uno de los habitantes de un país, a pesar de que el objeto y la magnitud de esos derechos sean escasamente determinables y difícilmente justiciables. Con respecto a este último punto se presenta una de las mayores dificultades para la ejecución de estos derechos, que puede enunciarse así: ¿qué ocurre cuando los gobiernos nacionales, provinciales o municipales dejan de satisfacer las demandas básicas, sea por corrupción, por negligencia o, por mera imposibilidad? La primera respuesta a este interrogante consiste en examinar si y en qué forma está abierta la vía jurídica para lograr que los derechos económicos y sociales sean efectivamente justiciables allí en donde los potenciales beneficiarios de los mismos estén privados de ellos.

Tomaré un ejemplo particularmente dramático de la situación argentina actual. Me refiero, en especial, a la exigencia de que el estado provea un seguro de desempleo para todos los ciudadanos que no encuentren temporaria o permanentemente trabajo. Tal derecho, estipulado en el art. 9. ${ }^{\circ}$ del Pacto internacional sobre derechos económicos y sociales, forma parte desde 1994 de la Constitución de la R. Argentina, pese a lo cual no se ha tomado ninguna medida legislativa para hacerlo efectivo, no solamente en momentos de crisis, como ocurrió desde el 2001 a la actualidad, sino tampoco en el momento de mayor afluencia de recursos al estado. ¿Por qué, en estas situaciones, no es posible obligar judicialmente a un estado a cumplir con las obligaciones contraídas mediante pactos internacionales? Por cierto, la objeción que habitualmente se hace a una exigencia como ésta se basa en la imposibilidad de atender a 
las necesidades de una gran mayoría de la población que padece la plaga del desempleo con los escasos recursos fiscales con que cuenta el gobierno federal, con los que debe atender otras obligaciones previamente contraídas. Si, prosigue la objeción, fuesen los jueces quienes dispusieran las prioridades en la distribución de los recursos presupuestarios, entonces estaríamos en una situación incompatible con la democracia, pues no serían los representantes del poder ejecutivo y los del congreso, elegidos por el pueblo, sino los jueces, que no es un poder directamente elegido por sus mandantes, los que se arrogarían la competencia para establecer las metas de una determinada política.

Una posible réplica a esta objeción está unida a una característica de la relación entre derechos y deberes a la que nos hemos referido antes. En efecto, como se ha señalado con acierto, los deberes asociados a determinados derechos se presentan no aislados sino en oleadas, es decir, conectados entre sí (cp. WALDRON, 1989: pp. 509511). Por consiguiente, conferir a los jueces una competencia para advertir a los otros dos poderes la existencia de deberes positivos incumplidos, que tienen, empero, rango de compromisos mediante tratados internacionales, y señalar que el cumplimiento de este deber está ligado a la efectiva realización de otros deberes, como por ejemplo, el de abstenerse de realizar otros gastos o de recortar ciertos impuestos con fines puramente electoralistas, no equivale a sustituir el ejercicio de la función política por la tiranía de los jueces, ya que el papel de éstos se limitaría a su función de fijar restricciones al margen de maniobra política que tienen los otros dos poderes, estableciendo en este caso qué metas deben ser prioritariamente alcanzadas por imperio constitucional, antes de disponer de la libertad de fijar otras por parte de los políticos.

De modo general, la tesis que sostenemos es la siguiente: la satisfacción de los derechos económicos y sociales, en tanto derechos positivos de los ciudadanos, implica necesariamente una restricción del margen de maniobra tanto en la imposición como en la distribución de los recursos fiscales y, en general, en la política económica del estado para los funcionarios políticos en ejercicio, ya que existen metas sociales prioritarias para la ciudadanía que deben ser satisfechas con anticipación a cualquier otra medida — como, por ejemplo, de rebaja de la tasa de impuestos a las ganancias, o de exención de impuestos a las rentas financieras- que los dirigentes tuvieran en su programa. En términos más abstractos, esto significa que, dada la interconexión de los deberes a la que nos hemos referido, se produce una importante consecuencia para la posible regulación y justiciabilidad de los derechos positivos de los ciudadanos, que se puede formular así: la primera obligación de quienes tienen los deberes positivos especiales de procurar satisfacer los derechos económicos y sociales de los ciudadanos, consiste en abstenerse de tomar cualquier medida que empeore previsiblemente la situación existente, ya precaria, de los peor situados. De este modo, así como los derechos negativos dan origen a la aparición de derechos positivos complementarios, que consisten en que se preserve la vida e integridad de los ciudadanos y dan lugar a deberes positivos especiales por parte de los funcionarios y gobernantes, tenemos ahora la figura inversa: los derechos positivos de los ciudadanos dan origen a la aparición de derechos negativos complementarios de éstos, que tienen un correlato, en este caso estricto, con unos deberes negativos específicos para aquellos que estén en situaciones de poder, fundamentalmente económico. Éstos consisten en refrenar toda acción de carácter económico o social que haga imposible el ejercicio de los derechos posi- 
tivos por parte de la gran mayoría de los ciudadanos, agravando situaciones ya de pr sí malas. Son estos deberes negativos complementarios los que, por su propia naturaleza, se prestan más a ser considerados justiciables por un tribunal, con el debido asesoramiento de expertos, el que debería conocer sobre aquellos casos cuyas consecuencias para el empobrecimiento generalizado de una población o el brusco empeoramiento de sus condiciones de vida sean previsiblemente graves, como por ejemplo ingentes maniobras financieras, vaciamiento de los fondos de la seguridad social, etc.

$\$ 6$. Podemos formular en este momento la pregunta crucial desde el punto de vista filosófico-jurídico con respecto a la intervención del máximo tribunal constitucional en pro de la satisfacción de los derechos económicos y sociales de los ciudadanos, a saber: ¿ están autorizados los jueces a apelar a principios sustantivos de carácter moral para exigir el cumplimiento de las demandas basadas en estos difusos derechos humanos? ¿No están en ese caso simplemente poniendo su propia interpretación filosófica y moral de la constitución por encima de la de los otros dos poderes? Antes de analizar las posibles respuestas a esta pregunta, consideremos un aspecto importante que hasta ahora hemos dejado de lado. En efecto, la exposición que antecede muestra cómo ambas especies de derechos, los civiles y políticos y los económicos y sociales, están estrecha e inseparablemente involucradas, pues para que pueda ser ejercida una demanda por un cumplimiento efectivo de los derechos económicos por parte del poder ejecutivo o legislativo, debe contarse con la garantía acordada por un poder judicial independiente que vele por la protección de los derechos civiles, como el de asociarse libremente, peticionar y demandar la protección de sus derechos, expresar y sostener sus ideas e informar a la opinión pública de la existencia de situaciones de extrema precariedad o indigencia, en un determinado país, región o provincia, etc. En resumen, la efectiva ejecución de los derechos económicos y sociales no solamente no debe ser un pretexto para soslayar el cumplimiento de una activa protección de los derechos civiles y políticos, como ocurre frecuentemente, sino que, al contrario, presupone que ambas especies de derechos están necesariamente en vigencia y deben ser simultáneamente respetados. Esta indisoluble conexión entre las dos especies de derechos humanos pone de manifiesto simultáneamente la verdadera naturaleza de ambos: no se trata de principios morales sustantivos que están por fuera y por encima del sistema — cmo para los pueblos jerárquicos no liberales ni democráticos- sino de las reglas de argumentación más generales que están en la base del sistema democrático.

Adoptaré como punto de partida la distinción que hace DwORKIN (1978: pp. 71 80) entre «reglas» y «principios», sistematizada más tarde por ALEXY (1986: pp. 75 79) y más recientemente por ATIENZA, según la cual toda norma regulativa es o una regla o un principio: «las reglas tienen condiciones de aplicación [...] cerradas, en el sentido de que consisten en una serie de propiedades [...] establecidas previamente, mientras que los principios obedecen más bien a lo que VON WRIGHT llamaba normas categóricas, esto es, normas que no tienen más condiciones de aplicación que las que surgen del propio contenido» (ATIENZA, 2006: pp. 218-234). Dentro de este marco, tendríamos un conflicto entre dos principios que forman parte del sistema jurídico: uno que afirma la primacía del poder legislativo, en tanto un cuerpo de representantes del pueblo elegidos de modo directo por éste a fin de llevar adelante una determinada política pública, sobre el poder judicial, compuesto por expertos con compe- 
tencia limitada y no elegidos directamente por sus mandantes, razón por la cual estos últimos deberían abstenerse de crear nuevas leyes (el argumento tiene varias versiones; adopto la que presenta DwORKIN, 1978: 84-85). El otro que sostiene que el derecho a la vida que todas las constituciones de los estados democráticos protegen, «no significa solamente que una persona no puede ser privada de su vida por la ejecución de una sentencia de muerte, salvo en el caso de que hubiera un procedimiento legal establecido por la ley. [...] Un aspecto igualmente importante de ese derecho es el derecho a que la vida sea viable, porque ninguna persona puede vivir sin los medios para ello. Si el derecho a que la vida sea posible no es tratado como una parte del derecho constitucional a la vida, el modo más fácil de privar a una persona de su derecho a la vida sería, entonces, privarla de sus medios de subsistencia» (Corte Suprema de la India, AIR 1986 Supreme Court 18, en: STEINER y Aston, 2000: p. 288).

Ahora bien, cuando dos principios entran en conflicto, el único modo de resolverlo es mediante una ponderación entre ambos que permita determinar cuál de los dos, todos los aspectos del caso considerados, debe obtener la primacía. Dado que, por su carácter, los principios son siempre principios prima facie, el hecho de que uno de los que colisionan entre sí deba ceder en ciertas circunstancias ante el otro no significa que el principio postergado pierda validez o importancia en general sino solamente para el caso en cuestión (HARE, 1981: 38-43; ATIENZA, 2006: 240-2).

¿Qué jerarquía debemos atribuirles a los dos principios enunciados en los arts. $1{ }^{\circ}$ y $3 .^{\circ}$ de la Declaración Universal de los DDHH, que afirman, respectivamente: «todos los seres humanos nacen libres e iguales en dignidad y derechos»; y «todos tienen derecho a la vida, a la libertad y a la seguridad de las personas»? Sin duda, se trata en primer lugar de principios morales universales cuya validez y vigencia debe extenderse a todo el planeta, razón por la que, como señalamos, establecen criterios de demarcación entre las sociedades democráticas liberales —en las que ya la tienen-y las que (aún) no lo son. Pero lo importante es que en las sociedades democráticas liberales no constituyen meros principios morales sino que éstos, desde la formación de las mismas como regímenes constitucionales, están establecidos y formalizados como derechos subjetivos fundamentales de los ciudadanos, a través de cuyo reconocimiento ellos se conceden recíprocamente el respeto propio de sujetos de pleno derecho (HABERMAS, 1992: 112-135; 1997: 221-225). Para los estados constitucionales de derecho, por lo tanto, estos derechos fundamentales deben ser entendidos como metarreglas formales del estado, las cuales reglamentan la legitimidad y preservación del régimen constitucional mismo. Su prioridad, entonces, sobre cualquier otro principio contrapuesto puede advertirse dialécticamente por el hecho de que la aserción de cada uno de dichos principios implica pragmáticamente el rechazo de la aserción del principio contradictorio, que permitiría un estado de cosas incompatible con una democracia constitucional. En efecto, es imposible admitir bajo ninguna circunstancia que uno cualquiera de los ciudadanos de un estado democrático naciese carente de libertad o de igualdad en dignidad y derechos, a diferencia de todos los demás (GUARIGLIA, 1996: pp. 162-169). Si, en consecuencia, las metarreglas constitutivas del estado democrático de derecho deben ser preservadas, se sigue, entonces, que ese estado deb brindar a sus ciudadanos las necesarias oportunidades tanto para preservar su vida, como señala el fallo de la Corte Suprema de la India, como para desarrollar su propia autonomía como miembros libres e iguales, para lo cual es necesario asegurarles no sola- 
mente medios mínimos de subsistencia sino también de desarrollo y formación que les permitan integrarse como un miembro activo de la sociedad, gozando de sus capacidades y habilidades, mientras sus fuerzas lo permitan, y de la seguridad de que se le proveerá de una atención condigna, cuando aquellas flaqueen. En resumen, los arts. 22 al 26 de la Declaración que enumeran los derechos humanos económicos y sociales más básicos que un estado de derecho debe satisfacer deben ser interpretados como una consecuencia en el plano conceptual de lo que significa ser un ciudadano libre e igual en una sociedad moderna, que cuenta con una sociedad civil ampliamente desarrollada y una economía de mercado. La intervención de los jueces en la preservación de las metarreglas constitutivas de esa sociedad como soporte del régimen político no es una intervención para llevar a cabo una política con una meta positiva, como si ésta hubiese sido elegida por una mayoría de los ciudadanos, lo que sería una injustificada intromisión en la esfera de los otros dos poderes, sino que, al contrario, es una actividad de vigilancia y preservación de las reglas constitutivas de esa sociedad mediante la salvaguarda del estatus de sus ciudadanos como miembros autónomos de la misma. Si la división de poderes propia de una forma republicana de gobierno es la regla marco del régimen político que permite preservarlo, entonces la función de los jueces como miembros independientes en función de árbitros, y en especial del tribunal constitucional supremo, es la de reinterpretar y reconstruir esas metarreglas cuando la acción u omisión de los otros dos poderes amenace con restringirlas, distorsionarlas o directamente cambiarlas sin recurrir al procedimiento de enmienda estipulado por la constitución isma.

\section{BIBLIOGRAFÍA Y REFERENCIAS}

AleXY, R., 1978: Theorie der juristischen Argumentation, Francfort: Suhrkamp.

- 1986: Theorie der Grundrechte, Francfort: Suhrkamp.

AtienzA, M., 2006: El derecho como argumentación, Barcelona: Ariel.

BeItZ, Ch., 2001: «Human Rights as a Common Concern», American Political Science Review, 95, pp. 269-282.

Center for the Study of Human Rights, 1994: Twenty-Five Human Rights Documents, Columbia University.

CoHen, J., 2004: «Minimalism About Human Rights: The Most We Can Hope For?», Journal of Political Philosopby, 12, pp. 190-213.

CROCKER, D., 1998: Florecimiento bumano y desarrollo internacional, San José: Editorial de la Universidad de Costa Rica.

De Greiff, P., y Cronin, C. (edit.), 2002: Global Justice and Transnational Politics, Cambridge, Mass.-London: The MIT Press.

Dworkin, R., 1978: Taking Rights Seriously, Londres: Duckworth, 2. ${ }^{a}$ ed.

FABRE, C., 1998: «Constitutionalising Social Rights», Journal of Political Philosophy, 6, pp. 263 284.

FinNIS, J., 1980: Natural Law and Natural Rights, Oxford: Clarendon Press.

Freeman, S., 2006: «The Law of Peoples, Social Cooperation, Human Rights, and Distributive Justice», Social Philosophy E Policy Foundations, 23, pp. 29-68.

GEWIRTH, A., 1982: Human Rights, Chicago \& Londres: University of Chicago Press.

- 1996: The Community of Rights, Chicago \& Londres: The University of Chicago Press. 
Guariglia, O., 1993: «El concepto normativo de "persona” y los requisitos mínimos de justicia distributiva en una sociedad democrática», Doxa (Cuadernos de Filosofía del Derecho), 13, Alicante: pp. 109-122.

- 1995: Universalismus und Neuaristotelismus in der zeitgenössischen Ethik, HildesheimZürich-New York: Georg Olms (Philosophische Texte und Studien, Bd. 40).

- 1996: Moralidad (Ética universalista y sujeto moral), Buenos Aires: Fondo de Cultura Económica (Biblioteca de Filosofía) [trad. ital. Nápoles, 2002].

- 2002: Una ética para el siglo xxi: Ética y derechos humanos en un tiempo posmetafísico, Buenos Aires-México: Fondo de Cultura Económica.

HABERMAs, J., 1992: Faktizität und Geltung, Francfort: Suhrkamp.

- 1996: Die Enbeziehung des Anderen, Francfort: Suhrkamp.

— 2002: «On Legitimation through Human Rights», en: DE GreIFF, P., y CrOnIN, C. (edit.), pp. 197-214.

Hare, R. M., 1981: Moral Thinking, Oxford: Clarendon Press.

KAnT, I., 1963: MS, Metaphysik der Sitten, en: Werke, edición de W. Weischedel, tomo IV, Darmstadt, Wissens, Buchgesell.

KeRSTING, W., 1993: Wohlgeordnete Freiheit (Immanuel Kants Rechts- und Staatsphilosophie), Francfort: Suhrkamp, 2. ${ }^{\text {a }}$ ed.

Maritain, J., 1953: L'bomme et l'état, Paris: Presses Universitaires de France.

NinO, C., 1989: Ética y derechos bumanos, 2." ed., Buenos Aires: Astrea.

NozICK, R., 1980: Anarchy, State, and Utopia, Oxford-Cambridge, Mass.: Blackwell.

O’NeILl, O., 2000: Bounds of Justice, Cambridge: Cambridge U.P.

Peces Barba, G. (comp.), 1989: El fundamento de los derechos humanos, Madrid: Debate. Pogge, T. (edit.), 2001: Global Justice, Oxford: Blackwell.

- 2002: World Poverty and Human Rights, Cambridge: Polity Press.

Rawls, J., 1993: Political Liberalism, Nueva York: Columbia University.

- 1999: The Law of Peoples, Cambridge, Mass.-Londres: Harvard University Press.

SEN, A., 1992: Inequality Reexamined, Cambridge, Mass.: Harvard University Press.

Shue, H., 1988: «Mediating Duties», Ethics, 98, pp. 687-704.

- 1996: Basic Rights, 2. ${ }^{a}$ ed., Princeton: Princeton U.P.

Steiner, H., y Alston, Ph., 2000: International Human Rights in Context: Law, Politics Morals, 2. ${ }^{a}$ ed., Oxford: University Press.

Stiglitz, J. E., 2002: Globalization and Its Discontents, Nueva York-Londres: W. W. Norton. WaldRON, J., 1989: «Rights in Conflict», Ethics, 99, pp. 503-519.

- 1993: Liberal rights, Cambridge: Cambridge U.P.

WAlZER, M., 1994: Thick and Thin: Moral Argument at Home and Abroad, Notre Dame-London: University of Notre Dame Press. 